\title{
ESPECIE NUEVA DE SANGUIJUELA DEL GÉNERO HELOBDELLA (RHYNCHOBDELLIDA: GLOSSIPHONIIDAE) DEL LAGO DE CATEMACO, VERACRUZ, MÉXICO
}

\author{
Alejandro Oceguera-FigueroA \\ City University of New York (CUNY), Graduate School and University Center y Division of \\ Invertebrate Zoology, American Museum of Natural History. \\ Central Park West at 79th Street, Nueva York, Nueva York, 10024, EUA. \\ aoceguera@amnh.org \\ RESUMEN
}

Se describe una especie nueva de sanguijuela del género Helobdella del Lago de Catemaco, Veracruz, México con base en 23 ejemplares. Los organismos se encontraron adheridos a piedras y raíces a las orillas del lago. La especie nueva carece de placa quitinoide dorsal y se diferencia del resto de las especies del género por presentar la superficie dorsal del cuerpo obscura con manchas blancas de tamaño y distribución muy variable; de tres a cinco hileras dorsales de papilas; glándulas salivales difusas en el parénquima; buche con seis pares de ciegos, el último par forma post-ciegos o divertículos.

Palabras Clave: Hirudinea, Glossiphoniidae, Helobdella, Catemaco, México, sanguijuela.

\section{ABSTRACT}

A new leech species of the genus Helobdella from Catemaco Lake, Veracruz, Mexico is described based on the examination of 23 specimens. Leeches were found attached to submerged rocks and plants. The new species lacks a nuchal scute and is distinguishable from other species of the genus by the presence of a obscure dorsal surface with white spots of different size and irregularly arranged; three or five longitudinal rows of dorsal papillae; salivary glands diffused in the parenchyma; six pairs of crop caeca, the posterior pair forming post-caeca or diverticula.

Key Words: Hirudinea, Glossiphoniidae, Helobdella, Catemaco, Mexico, leech.

\section{INTRODUCCIÓN}

El género Helobdella incluye especies de sanguijuelas dulceacuícolas que se caracterizan por presentar en la mayoría de los casos el cuerpo aplanado dorsoventralmente, un par de manchas oculares cefálicas, un anillo que separa los gonoporos y por carecer tanto de micetomas como de órganos esofágicos (Siddall \& Borda 2003). La mayoría de las especies del género se alimentan de invertebrados acuáticos como moluscos y oligoquetos y no existen representantes hematófagos. Presentan una distribución mundial con excepción de la Antártica, con la mayor riqueza de especies en América del Sur. Sawyer (1986) dividió al género Helobdella en dos series: stagnalis que incluye especies con placa quitinoide dorsal y triserialis para las especies que carecen 
de dicha placa. Estudios recientes sobre las relaciones filogenéticas del grupo han puesto en duda la validez de dicho caracter para reconocer grupos naturales (Light \& Siddall 1999; Siddall \& Borda 2003). En tales estudios se ha demostrado que varias características morfológicas (e.g. glándulas salivales compactas y número de ciegos en el buche) empleadas por Ringuelet (1978a) para subdividir al género Helobdella en cinco géneros (Dacnobdella, Acritobdella, Adaetobdella, Gloiobdella y Helobdella) no definen grupos naturales y por lo tanto se considera a Helobdella como el único género válido (Siddall \& Borda 2003).

En México se han registrado dos especies de Helobdella con placa quitinoide dorsal (Oceguera-Figueroa \& León-Règagnon 2005): Helobdella stagnalis (Linnaeus 1758) y Helobdella atli Oceguera-Figueroa \& León-Règagnon 2005 y tres especies sin placa quitinoide dorsal (Ringuelet 1981): Helobdella elongata (Castle 1900), Helobdella conchata (Caballero 1941) y Helobdella triserialis (Blanchard 1849). Las especies Helobdella socimulcensis (Caballero 1931), Helobdella moorei Caballero 1933, y los registros de Helobdella fusca (Castle, 1900) realizados por Oka (1932) y Caballero $(1935,1940)$ son considerados como H. triserialis por Ringuelet (1985) y Sawyer (1986). Recientemente se ha sugerido, con base en hipótesis filogenéticas, que los registros de Norteamérica (EUA) de Helobdella triserialis y Helobdella stagnalis deben ser re-asignados a las especies Helobdella papillata (Moore 1906) y Helobdella modesta (Verrill, 1872), respectivamente (Siddall \& Borda 2003; Siddall et al. 2005). Sin embargo, al no incluir ejemplares recolectados en México, se considera que tanto $H$. triserialis como $H$. stagnalis se distribuyen en México, al menos hasta que se realice un estudio formal de las mismas.

Durante la recolecta de ejemplares de hirudíneos en el lago de Catemaco, Veracruz, México, se detectó una especie nueva del género Helobdella, cuya descripción morfológica se realiza en el presente trabajo.

\section{MATERIAL Y MÉTODOS}

Las sanguijuelas se encontraron adheridas a raíces y debajo de piedras a las orillas del lago de Catemaco, Veracruz, México. (permiso de colector científico FAUT0056 otorgado por la SEMARNAT). Los ejemplares se capturaron con la mano y se transportaron al laboratorio en recipientes de plástico con agua del medio, donde se procedió a anestesiarlos mediante la adición gradual de alcohol al $70 \%$. Posteriormente fueron fijados en formol al $4 \%$ o en alcohol absoluto. Las observaciones, disecciones y esquemas se realizaron con un microscopio estereoscópico (Zeiss modelo 475052). Las microfotografías de los ejemplares fueron tomadas con una cámara digital Olympus (Camedia C-3040 Zoom) adaptada a un microscopio. Los ejemplares tipo se depositaron en la Colección Nacional de Helmintos (CNHE), Instituto de Biología, Universidad Nacional Autónoma de México, México, D.F., y en la colección de anélidos del American Museum of Natural History, (AMNH), Nueva York, EUA. 


\section{RESULTADOS}

\section{Helobdella virginiae sp.nov. Figuras 1-6}

La descripción se realizó con base en 23 ejemplares (dos preparaciones permanentes, dos disecciones y 19 ejemplares completos).

\section{Morfología externa}

Sanguijuelas pequeñas, 5.7-9.6 mm de largo por 2.7-4.9 mm de ancho. Los adultos presentan la superficie dorsal obscura con manchas blancas, de número y disposición muy variable (Fig. 1). En la mayoría de los ejemplares la superficie ventral es blanca o marrón con pigmentos obscuros que no siguen ningún tipo de patrón (Fig. 2). Superficie dorsal con 3-5 hileras longitudinales de papilas en a2 de cada somito, muy notorias en la región media y posterior del cuerpo, una de las hileras es media y una o dos a cada lado, algunos ejemplares carecen de papilas evidentes. Superficie ventral sin papilas. Gonoporos separados por un anillo, masculino en XII a1/a2 y femenino en XII a2/a3. Un par de manchas oculares en IV, bien separadas entre sí (Fig. 3). Carecen de placa quitinoide dorsal. Ano localizado en la superficie dorsal entre los somitos XXVI y XXVII. Somitos I y II fusionados, somito III unianillado, Somitos IV y V bianillados. Somitos VI-XXIV triananillados. Somitos XXV y XXVI bianillados. Somito XXVII unianillado. Ventosa anterior más o menos triangular. Boca en la región anterior de la ventosa, subterminal. Ventosa posterior circular.

\section{Morfología interna}

Proboscis envuelta en una cubierta membranosa. Células salivales dispersas en el parénquima entre XI-XVI. Los conductos de las células salivales se unen y forman un conducto común que se inserta en la base de la proboscis en el somito XI. Esófago simple, no recurvado. Buche con seis pares de ciegos de XIV a XIX, el último de ellos con recorrido descendente, formando postciegos o divertículos que alcanzan el somito XXIV. Intestino con cuatro pares de ciegos de XX a XXIII (Fig. 4). Seis pares de testisacos intersegmentales de XIII/XIV-XVIII/XIX, el primer par poco desarrollado. Vasos deferentes simples muy largos, sin recorrido ascendente, con recorrido descendente hasta el somito XX (Fig. 5). Ovisacos simples que alcanzan el somito XVII (Fig. 6).

\section{Material examinado}

Holotipo. (CNHE 5474) Fijado en Formol 4\%, Conservado en una mezcla de glicerina, alcohol y formol. $8.3 \mathrm{~mm}$ largo, $3.6 \mathrm{~mm}$ ancho. MÉXICO. Lago de Catemaco, Colonia "La Victoria", Veracruz. $18^{\circ} 22^{\prime} 33.4^{\prime \prime}$ N, 95 06' 34.4" O. Altitud 335 m. Recolectado por A. Oceguera-Figueroa, E. Martínez, L. Romero, R. Bautista y A. Espinoza. 9 Septiembre 2005. Paratipos. (CNHE 5475, 5476) 19 ejemplares, (AMNH Annelida 5326) 4 ejemplares. Misma localidad, recolectores y fecha que el holotipo.

Etimología: El epíteto específico "virginiae" hace referencia a la Dra. Virginia León Règagnon, en reconocimiento a sus investigaciones en el campo de la helmintología.

\section{DISCUSIÓN}

De acuerdo con la diagnosis del género Helobdella, los miembros del mismo presentan los gonoporos separados por un solo anillo, un par de manchas oculares cefálicas y 
Oceguera-Figueroa: Especie nueva de sanguijuela de Catemaco, Veracruz
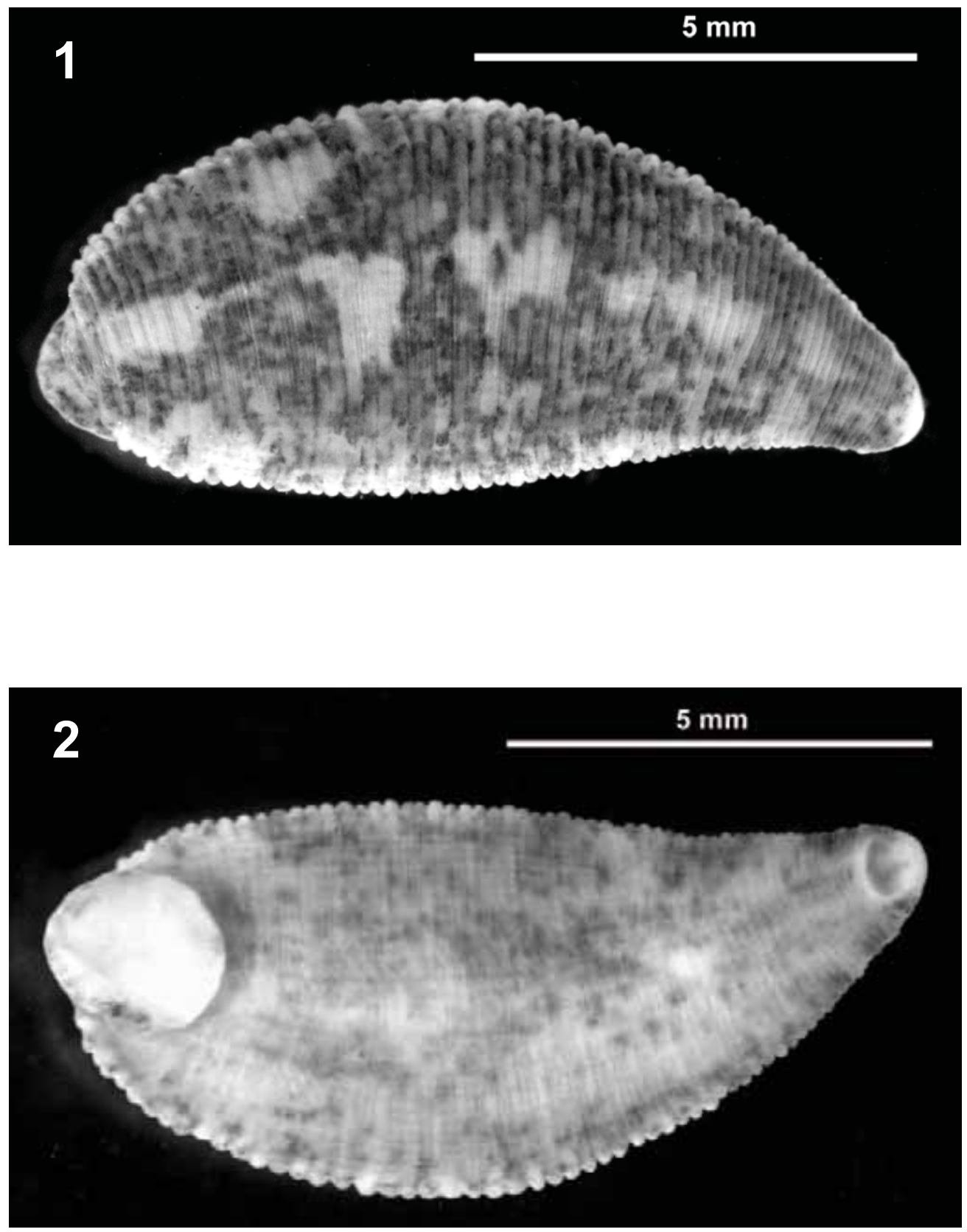

Figuras 1-2

Helobdella virginiae sp. nov. Holotipo 1) Vista dorsal, 2). Vista ventral. 

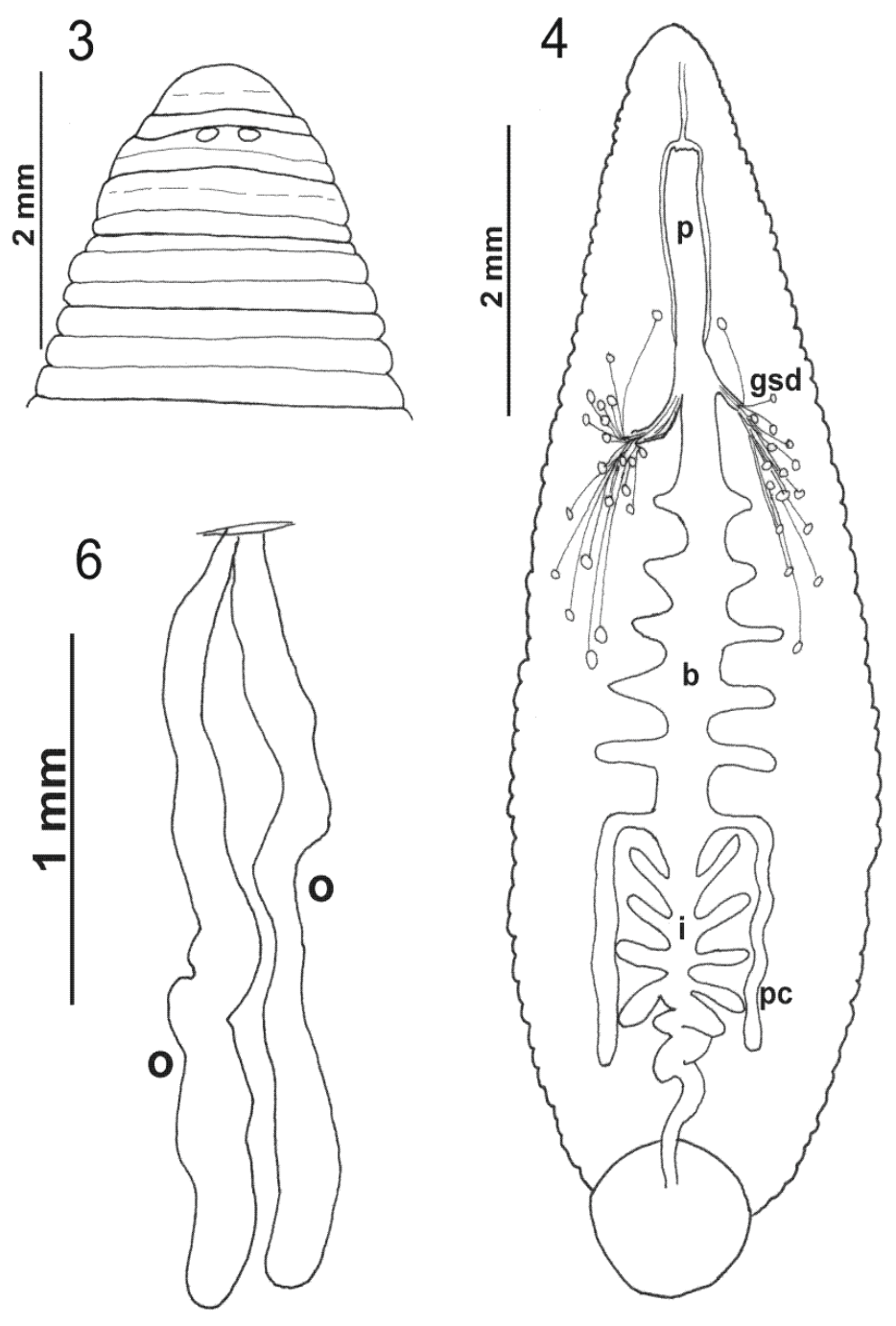

5

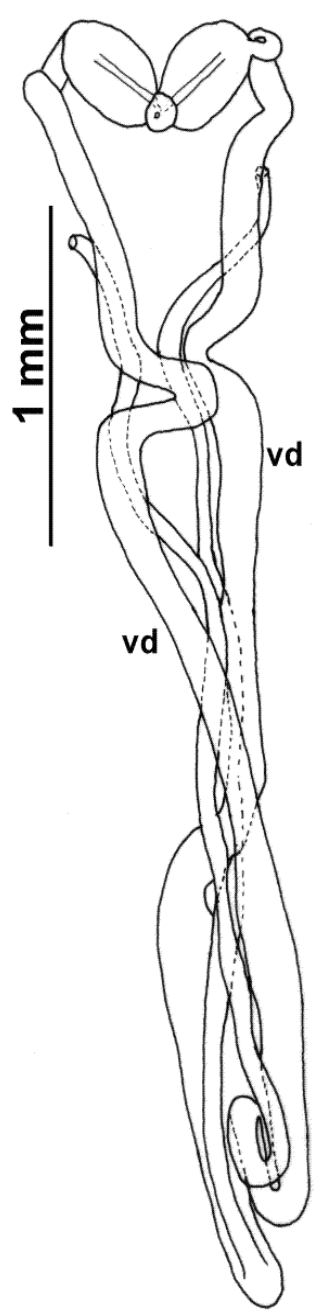

Figuras 3-6

Helobdella virginiae sp. nov. 3) Detalle de la región anterior.4) Aparato digestivo. p- proboscis; gsdglándulas salivales difusas; b- buche; pc- post-ciegos; i- intestino.5) Aparato reproductor masculino. vdVasos deferentes.6) Aparato reproductor femenino. o- ovisacos. 
carecen de órganos esofágicos y micetomas. Estos caracteres son consistentes con los de la especie que aquí se describe, por lo cual es claro que se trata de una especie del género Helobdella. Helobdella virginiae se distingue fácilmente del resto de sus congéneres por presentar el dorso obscuro con manchas blancas distribuidas irregularmente, y por las hileras longitudinales de papilas en la región dorsal.

En el continente Americano, este género se encuentra ampliamente distribuido; para Norteamérica se han descrito siete especies carentes de placa quitinoide dorsal (Klemm 1982; Ringuelet 1981; Sawyer 1986; Siddall \& Borda 2003): Helobdella elongata carece de ciegos en el buche a excepción de post-ciegos o divertículos y el cuerpo es alargado, angosto, carece papilas y pigmentos, mientras que $H$. virginiae presenta cinco pares de ciegos en el buche y un par de post-ciegos, cuerpo aplanado dorsoventralmente así como pigmentos y papilas. Helobdella triserialis, Helobdella robusta Shankland, Bissen \& Weisblat 1992 y Helobdella lineata Moore 1952, se diferencian de $H$. virginiae, ya que presentan numerosas hileras longitudinales de pigmentos obscuros mientras que la última presenta la región dorsal obscura con manchas blancas distribuidas irregularmente. Helobdella virginiae es fácilmente distinguible de $H$. conchata, ya que esta última presenta los anillos subdivididos mientras que $H$. virginiae presenta tres anillos no subdivididos por cada somita completa. Helobdella transversa Sawyer 1972 tiene franjas transversales de manchas blancas, estas se encuentran en el anillo a2 de cada somita, formando un patrón completamente distinto al de $H$. virginiae, que carece de franjas transversales. Helobdella fusca presenta una gran variación en los patrones de pigmentación de la superficie dorsal (Klemm 1982), Helobdella virginiae es muy parecida a la forma señalada por Klemm (1982) como "scattered pigmented form», sin embargo, es fácilmente diferenciable de $H$. virginiae, ya que $H$. fusca carece de cualquier tipo de papilas en la superficie dorsal, además de presentar manchas blancas en la región anal (anal patches), mientras que $H$. virginiae presenta tres o cinco hileras longitudinales de papilas en la superficie dorsal y carece de manchas blancas en la región anal de manera constante.

En América del Sur se encuentra la mayor diversidad de especies del género Helobdella (Ringuelet 1985, Siddall \& Borda 2003). Hasta la fecha se han registrado aproximadamente 25 especies sin placa quitinoide dorsal en esa región del continente (Ringuelet 1985, Siddall 2001; Siddall \& Borda 2003, 2004).

Helobdella virginiae puede diferenciarse de Helobdella michaelseni Blanchard 1900, Helobdella obscura Ringuelet 1942, Helobdella wodzickiorum Siddall \& Borda 2004 y Helobdella similis Ringuelet 1942, ya que $H$. virginiae presenta cinco pares de ciegos simples en el buche y un par de postciegos o divertículos, mientras que $H$. michaelseni y $H$. obscura carecen de ciegos en el buche y $H$. similis y $H$. wodzickiorum únicamente presentan un par de post-ciegos o divertículos.

Las especies Helobdella cryptica (Ringuelet 1978), Helobdella chaquensis (Ringuelet 1978), Helobdella dubia (Ringuelet 1958), Helobdella longicolis Weber 1915, Helobdella xenoica (Ringuelet 1975), Helobdella titicacensis Ringuelet 1959, Helobdella malvinensis (Ringuelet 1978) y Helobdella sorojchi Siddall 2001 presentan glándulas salivales compactas, mientras que $H$. virginiae tiene glándulas salivales difusas en el parénquima. 
Helobdella villarsi Weber 1916 presenta los gonoporos en los anillos, mientras que $H$. virginiae presenta los gonoporos en los surcos interanulares. Helobdella paraguayensis Weber 1915, Helobdella chilensis Blanchard 1900, Helobdella peruvensis Weber 1916 y Helobdella huaroni Weber 1916 presentan dos anillos entre los gonoporos; por el contrario, $H$. virginiae tiene un solo anillo entre los gonoporos. La presencia de dos anillos entre los gonoporos en las especies señaladas no corresponde con la definición del género propuesta por Siddall \& Borda (2003); sin embargo, es probable que se trate de observaciones no muy precisas que deben ser tomadas con precaución. Independientemente de este carácter dudoso, $H$. paraguayensis y $H$. chilensis presentan la faringe recurvada y no recta como $\mathrm{H}$. virginiae. Helobdella peruvensis presenta siete líneas dobles longitudinales obscuras y $\mathrm{H}$. huaroni el dorso gris claro uniforme o pardo obscuro, características que no corresponden con la superficie obscura con manchas blancas de $H$. virginiae.

Helobdella ampullariae Ringuelet 1945, Helobdella festai (Dequal 1916), Helobdella hyalina Ringuelet 1942, Helobdella cordobensis (Ringuelet 1942) y Helobdella araucana Ringuelet 1978 carecen de papilas en la superficie dorsal, mientras que $H$. virginiae presenta tres o cinco hileras longitudinales dorsales de papilas. En Helobdella simplex (Moore 1911) y Helobdella brasilensis (Weber 1915) hay papilas dorsales en los tres anillos de cada somita, formando hileras longitudinales continuas, mientras que en $H$. virginiae, las papilas dorsales se encuentran exclusivamente en los anillo a2 de cada somita, formando hileras longitudinales con las papilas separadas entre sí por dos anillos. Helobdella striata (Ringuelet 1943) y Helobdella triserialis presentan un número variable de hileras longitudinales pigmentadas y de papilas, características que no concuerdan con las descritas para $H$. virginiae.

La descripción de una nueva especie de Helobdella para México nos hace suponer que su historia biogeográfica es mas compleja de lo que previamente se había considerado. El género Helobdella es de origen Suramericano (Ringuelet 1978b, Siddall et al 2005) y se dispersó hacia Norteamérica después de la formación del Istmo de Panamá hace aproximadamente 1.2 millones de años en más de un evento. Las especies del género Helobdella presentan un patrón biogeográfico similar al de las especies del género hematófago Haementeria (Oceguera-Figueroa 2006a; Ringuelet 1985) y al de las especies macrófagas del género Semiscolex (Oceguera-Figueroa [2006b]; Borda \& Siddall 2004).

\section{AGRADECIMIENTOS}

A Rosario Mata López y Florencia Bertoni por su ayuda en la obtención de las microfotografías y revisión del manuscrito. A Elizabeth Martínez, Luz Romero, Rocío Bautista y Arlette Espinoza por su ayuda en la recolección de ejemplares. A Luis García Prieto y Mark E. Siddall por su ayuda en el manejo de las colecciones biológicas. Este trabajo fue financiado por el proyecto NSF 0119329 de J. Campbell (UTA) y V. León-Règagnon (UNAM) y por CONACyT por medio de una beca de estudios de Posgrado del autor (172322). 
Oceguera-Figueroa: Especie nueva de sanguijuela de Catemaco, Veracruz

\section{LITERATURA CITADA}

Borda, E. \& M.E. Siddall. 2004. Arhynchobdellida (Annelida: Oligochaeta: Hirudinida): phylogenetic relationships and evolution. Mol. Phylo. Evol. 30:213-225.

Caballero, C.E. 1935. Hirudíneos de México. XI. An. Inst. Biol. Univ. Nac. Autón. Méx. 6:49-52. . 1940. Sanguijuelas del lago de Pátzcuaro y descripción de una nueva especie, Ilinobdella patzcuarensis. XIV. An. Inst. Biol. Univ. Nac. Autón. Méx. 11:449-464.

Klemm, D.J. 1982. Leeches (Annelida: Hirudinea) of North America. Aquatic Biology Section, Environmental Monitoring and Support Laboratory, Office of Support Laboratory. Office of Research and Development, U.S. Environmental Protection Agency. Cincinati. Ohio.

Light, J.E. \& M.E. Siddall. 1999. Phylogeny of the leech family Glossiphoniidae based on mitochondrial gene sequences and morphological data. J. Parasitol. 85:815-823.

Oceguera-Figueroa, A. 2006a. A new species of freshwater leech of the genus Haementeria (Annelida: Glossiphoniidae) from Jalisco State, Mexico. Zootaxa, 1110:39-45. . (2006b). A new leech species of Semiscolex (Arhynchobdellida: Semiscolecidae) from Catemaco lake, Veracruz, Mexico. Rev. Mex. Biodiv. 77: 199-203.

Oceguera-Figueroa, A. \& V. León-Règagnon. 2005. A new freshwater leech species of Helobdella (Annelida: Glossiphoniidae) from central Mexico. Zootaxa. 976:1-8.

Oka, A. 1932. Hirudinées extraeuropéennes du Musée Zoologique Polonais. Ann. Mus. Zool. Pol. 9:313-330.

Ringuelet, R.A. 1978a. Nuevos géneros y especies de Glossiphoniidae sudamericanos basados en caracteres ecto y endosomáticos (Hirudinea Glossiphoniiformes). Limnobios. 1:269-276.

- 1978b. Biogeografía de los hirudíneos de América del Sur y de Mesoamérica. Obra Cent. Mus. La Plata 6:1-27.

. 1981. Clave para el reconocimiento de los hirudíneos de México. An. Inst. Biol. Univ. Nal. Autón. de Méx., Ser, Zool. 52:89-97.

. 1985. Fauna de agua dulce de la Republica de Argentina. Hirudinea. Fundación para la educación, la ciencia y la cultura. Buenos Aires.

Sawyer, R.T. 1986. Leech biology and behaviour. Oxford University Press, Oxford.

Siddall, M.E. 2001. Hirudinea from the Apolobamba in the Bolivian Andes, Including Three New Species of Helobdella (Clitellata: Hirudinea). Am. Mus. Novitat. 3341:1-14.

Siddall, M.E. \& E. Borda. 2003. Phylogeny and revision of the leech genus Helobdella (Glossiphoniidae) based on mitochondrial gene sequences and morphological data and a special consideration of the triserialis complex. Zool. Scr. 32:23-33.

. 2004. Leech Collections from Chile including Two New Species of Helobdella (Annelida: Hirudinida). Am. Mus. Novitat. 3457: 1-18.

Siddall, M.E., R.B. Budinoff \& E. Borda. 2005. Phylogenetic evaluation of systematics and biogeography of the leech family Glossiphoniidae. Invertebr. Syst. 19: 105-112.

Recibido: 27 de junio 2006

Aceptado: 8 de diciembre 2006 